\title{
The effect of near-zone preconditioning on electromagnetic integral equations of first and second kind
}

\author{
O. Wiedenmann and T. F. Eibert \\ Technische Universität München, Lehrstuhl für Hochfrequenztechnik, Arcisstr. 21, 80290 Munich, Germany \\ Correspondence to: O. Wiedenmann (oliver.wiedenmann@tum.de)
}

\begin{abstract}
The linear equation systems which arise from the discretization of surface integral equations are conveniently solved with iterative methods because of the possibility to employ fast integral methods like the Multilevel Fast Multipole Method. However, especially integral equations of the first kind often lead to very ill-conditioned systems, which require the usage of effective preconditioners. In this paper, the regularization property of near-zone preconditioning operators on the Electric Field Integral Equation is demonstrated and investigated for problems of different size. Furthermore, comparisons are drawn to second-kind integral equations such as the Combined Field Integral Equation.
\end{abstract}

\section{Introduction}

Boundary integral equations are widely used for the solution of electromagnetic scattering and radiation problems, mainly because they require only the discretization of the surfaces of the involved objects and the radiation condition is imposed implicitly. By employing the Method of Moments (MoM) discretization, the corresponding integral operators are transformed into linear algebraic systems of equations (Rao et al., 1982). In combination with fast integral solvers like the Multilevel Fast Multipole Method (MLFMM) (Coifman et al., 1993), the solution and storage complexity can be reduced considerably. The MLFMM accelerates matrix-vector products by computing far-range interactions in a hierarchical manner, so that only matrix elements related to near-range interactions have to be computed explicitly. If this concept is embedded in an iterative Krylov Subspace Solver, even large scale problems can be solved with reasonable computational means.

However, the resulting linear algebraic systems are often ill-conditioned. Fredholm integral equations of the first kind such as the Electric Field Integral Equation (EFIE), where the unknown quantity only appears inside the integral, result in system matrices with large condition numbers due to the unbounded integral operators. The Magnetic Field Integral Equation (MFIE) shows better convergence behavior, since it is an integral equation of the second kind with a compact integral operator, but it can only be formulated for objects with closed surfaces and shows worse solution accuracy due to the difficulty of discretizing the identity operator accurately. The Combined Field Integral Equation (CFIE) is a linear combination of EFIE and MFIE and inherits the characteristics of both of them. Additionally, it cures the problem of spurious solutions that can exist when one of the integral equations is solved independently. The CFIE exhibits worse solution accuracy than the pure EFIE and it is restricted to closed objects. On the other side, it shows a better convergence behavior than the EFIE. Its particular properties can be adjusted with the choice of the combination coefficient weighting the contributions of EFIE and MFIE (Yan et al., 1993).

In order to solve the resulting linear algebraic equation systems, sophisticated preconditioning concepts are required to achieve robust convergence. A particularly attractive technique is the inner-outer iterative approach (Saad, 1993), which employs an iterative preconditioner in an inner iteration loop. This method improves the convergence substantially while lowering the storage requirements for the basis vectors of the Krylov subspace. It is furthermore possible to extend this concept to a recursive iterative solution scheme, which can work with approximations of the system matrix in the inner preconditioning loops (Eibert, 2007).

If the MLFMM is employed, the near-zone matrix is particularly suited for the construction of algebraic preconditioners, since it contains the strongest interactions and it is explicitly available. Commonly used near-range preconditioners are Gauss-Seidel, incomplete LU or sparse approximate inverse approaches. Especially for the solution of first-kind integral equations, near-zone preconditioners can 
considerably improve the convergence (Wiedenmann and Eibert, 2011), so that even a complete LU decomposition of the near-zone matrix can be beneficial regarding the overall computation time (Wiedenmann and Eibert, 2012). In this case, the factorization of the near-field matrix leads to a regularization of the first-kind integral equation which is similar to a transformation into a second-kind integral equation in terms of convergence behavior. Another way of doing this is the use of Calderón-based preconditioners. Here, the self-regularizing property of the EFIE operator is exploited (Andriulli et al., 2008). However, the implementation of preconditioners based on the Calderón identity is a complex task and leads to several disadvantages. For instance, the discretization of the outer integral operator cannot be performed with the commonly employed Rao-WiltonGlisson (RWG) basis functions. Instead it requires the usage of Buffa-Christiansen basis functions (Buffa and Christiansen, 2007) or other basis functions with similar properties and in particular also the same disadvantages. The usage of these methods is often considered if the low-frequency breakdown of the EFIE needs to be resolved, too.

In this work, it shall be shown that a factorization of the near-zone matrix improves the convergence properties of the EFIE for small and medium-sized problems considerably so that it behaves like a second kind integral equation. Based on numerical results, it is demonstrated how the convergence characteristics change with growing problem size and how it compares with CFIE computations. Finally, the regularization property of a "perfect" near-zone preconditioner is investigated and explained in theory.

\section{Integral equation formulation}

Consider an impenetrable, perfectly electrically conducting object with a surface $A$ and an outwardly pointing unit normal vector $\boldsymbol{n}$. If the object is illuminated by an incident plane wave ( $E^{\mathrm{inc}}, H^{\mathrm{inc}}$ ), according to Love's equivalence principle the solution can be formulated in terms of equivalent electric surface currents $J_{A}$, which are defined on a so-called Huygens surface. By enforcing tangential boundary conditions for the electric fields, the integral equation for the electric field (EFIE) can be derived as

$$
\begin{aligned}
-\boldsymbol{n}(\boldsymbol{r}) & \times \boldsymbol{E}^{\mathrm{inc}}(\boldsymbol{r})=\boldsymbol{n}(\boldsymbol{r}) \\
& \times\left[j k Z \iint_{A}\left(\bar{I}+\frac{1}{k^{2}} \nabla \nabla\right) G\left(\boldsymbol{r}, \boldsymbol{r}^{\prime}\right) \boldsymbol{J}_{A}\left(\boldsymbol{r}^{\prime}\right) \mathrm{d} A^{\prime}\right]
\end{aligned}
$$

where $G\left(\boldsymbol{r}, \boldsymbol{r}^{\prime}\right)=\exp \left(-j k\left|\boldsymbol{r}-\boldsymbol{r}^{\prime}\right|\right) / 4 \pi\left|\boldsymbol{r}-\boldsymbol{r}^{\prime}\right|$ is the scalar Green's function, $Z$ the characteristic impedance and $k$ the wave number, all of free space. In a similar way, the MFIE can be formulated if boundary conditions for the tangential magnetic fields are imposed resulting in

$$
\begin{aligned}
-\boldsymbol{n}(\boldsymbol{r}) \times \boldsymbol{H}^{\mathrm{inc}}(\boldsymbol{r}) & =-\frac{1}{2} \boldsymbol{J}_{A}(\boldsymbol{r})+\boldsymbol{n}(\boldsymbol{r}) \\
& \times\left[\iint_{A} \nabla G\left(\boldsymbol{r}, \boldsymbol{r}^{\prime}\right) \times \boldsymbol{J}_{A}\left(\boldsymbol{r}^{\prime}\right) \mathrm{d} A^{\prime}\right] .
\end{aligned}
$$

To avoid the problem of interior resonances, the Combined Field Integral Equation (CFIE), which is a convex combination of Eqs. (1) and (2), can be formulated as

$\alpha \mathrm{EFIE}+Z(1-\alpha) \boldsymbol{n}(\boldsymbol{r}) \times \mathrm{MFIE}$

with the combination coefficient $\alpha$. In order to discretize the integral equations, the method of moments is employed with RWG basis and testing functions (Rao et al., 1982), leading to a linear algebraic system of equations of the form

$\mathbf{Z I}=\boldsymbol{V}$.

Here, $\mathbf{Z}$ is the fully populated impedance matrix, $\boldsymbol{V}$ the excitation vector and $\boldsymbol{I}$ the vector that contains the unknown coefficients of the expansion functions $\beta_{n}$ which approximate the total surface current $\boldsymbol{J}_{A}$ according to

$\boldsymbol{J}_{A}(\boldsymbol{r})=\sum_{n=1}^{N} I_{n} \beta_{n}(\boldsymbol{r})$

In order to reduce computational and memory cost, far-range interactions are computed in a hierarchical manner by the MLFMM. This reduces the complexity of a matrix-vector product from $\mathcal{O}\left(N^{2}\right)$ to $\mathcal{O}(N \log N)$ by splitting it into two parts: near-range interactions, which are computed explicitly and stored in $\mathbf{Z}_{\text {near }}$, and far-range interactions which are evaluated by the MLFMM. If we denote the MLFMM contribution as hypothetical matrix $\mathbf{Z}_{\mathrm{far}}$, the impedance matrix can be written as

$\mathbf{Z}=\mathbf{Z}_{\text {near }}+\mathbf{Z}_{\text {far }}$.

Due to the fact that $\mathbf{Z}_{\text {near }}$ is explicitly available and that it contains the strongest interactions, it is very well suited for the construction of near-zone preconditioners, which are widely used. Computing the inverse of the near-zone matrix and applying it to Eq. (4) yields

$$
\left(\mathbf{1}+\mathbf{Z}_{\text {near }}^{-1} \mathbf{Z}_{\text {far }}\right) \boldsymbol{I}=\mathbf{Z}_{\text {near }}^{-1} \boldsymbol{V},
$$

which represents the best possible algebraic near-zone preconditioner. In order to exploit the sparsity of the near-field matrix as far as possible, a LU decomposition of the nearfield matrix is preferred over the computation of the inverse, which, in general, is fully populated. It should be noticed that the application of the near-zone preconditioner introduces the identity operator 1, so that Eq. (7) has a similar form as the MFIE Eq. (2) and the CFIE Eq. (3). However, 

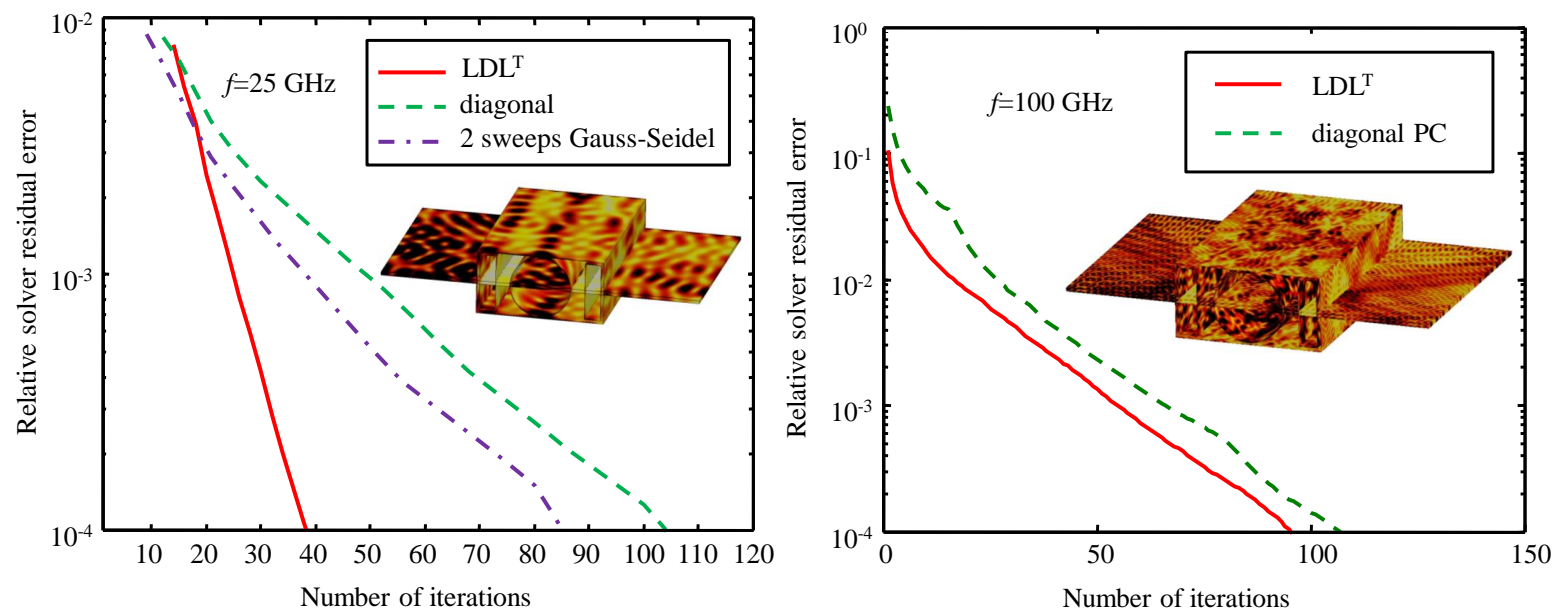

Fig. 1. Relative residual error for two PEC objects of different size: 196653 unknowns (left) and 1091335 unknowns (right).
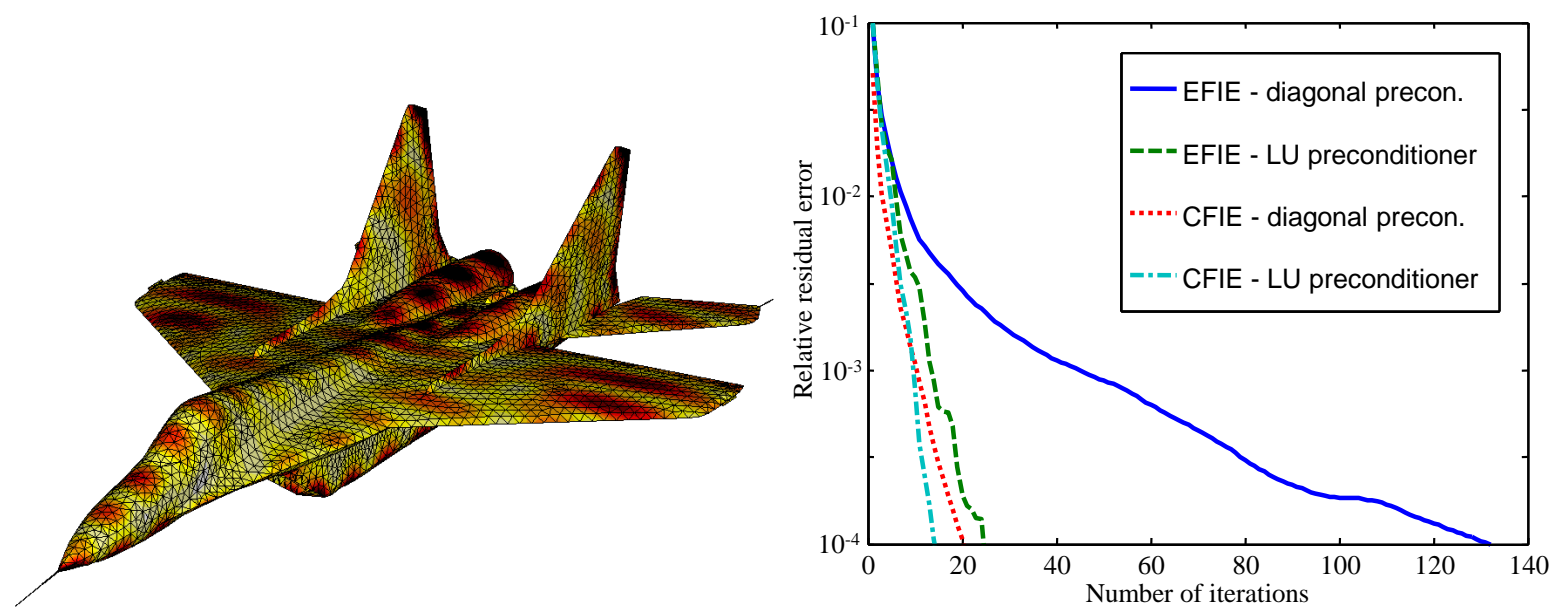

Fig. 2. Relative residual error for scattering computations on an air plane: comparisons between EFIE and CFIE computations and different near-zone preconditioners.

for growing problem size, the term $\mathbf{Z}_{\text {near }}^{-1} \mathbf{Z}_{\text {far }}$ is dominating and determines the convergence properties of the system for EFIE as well as for CFIE formulations. Moreover, if very high frequencies are considered, where many real resonances of the involved objects may exist, the systems become very ill-conditioned and the convergence deteriorates in all integral equation formulations. This behavior can generally not be cured by near-zone preconditioners.

In the following, we investigate the convergence characteristics of near-zone preconditioned EFIE formulations and second-kind integral equations such as CFIE for problems of different size.

\section{Numerical Results}

In this section, we compute the radar cross section (RCS) for various objects of different size. For the solution of the linear system, a flexible generalized minimum residual (FGMRES) solver with Given's rotations is used and a relative residual error of $10^{-4}$ in the outer loop is set as stopping criterion. To avoid the necessity of restarting the outer loop, an innerouter approach is employed, which uses the full matrix in both solver loops. The number of iterations in the inner loop is increased adaptively, depending on the decline of the residual in the outer loop. As an alternative to the LU decomposition preconditioner, weak near-zone preconditioners like Jacobi or Gauss-Seidel are implemented as well. If these weak near-zone preconditioners come to use, a second inner preconditioning loop is activated, which consists of another ten iterations with the explicitly computed near-zone elements.

As a first example, the RCS of a complicated structure is computed using the EFIE at a frequency of $25 \mathrm{GHz}$. The scattering object is discretized using 196653 unknowns and the convergence characteristics are shown in Fig. 1. It can be noticed that the LU decomposition preconditioner improves the 

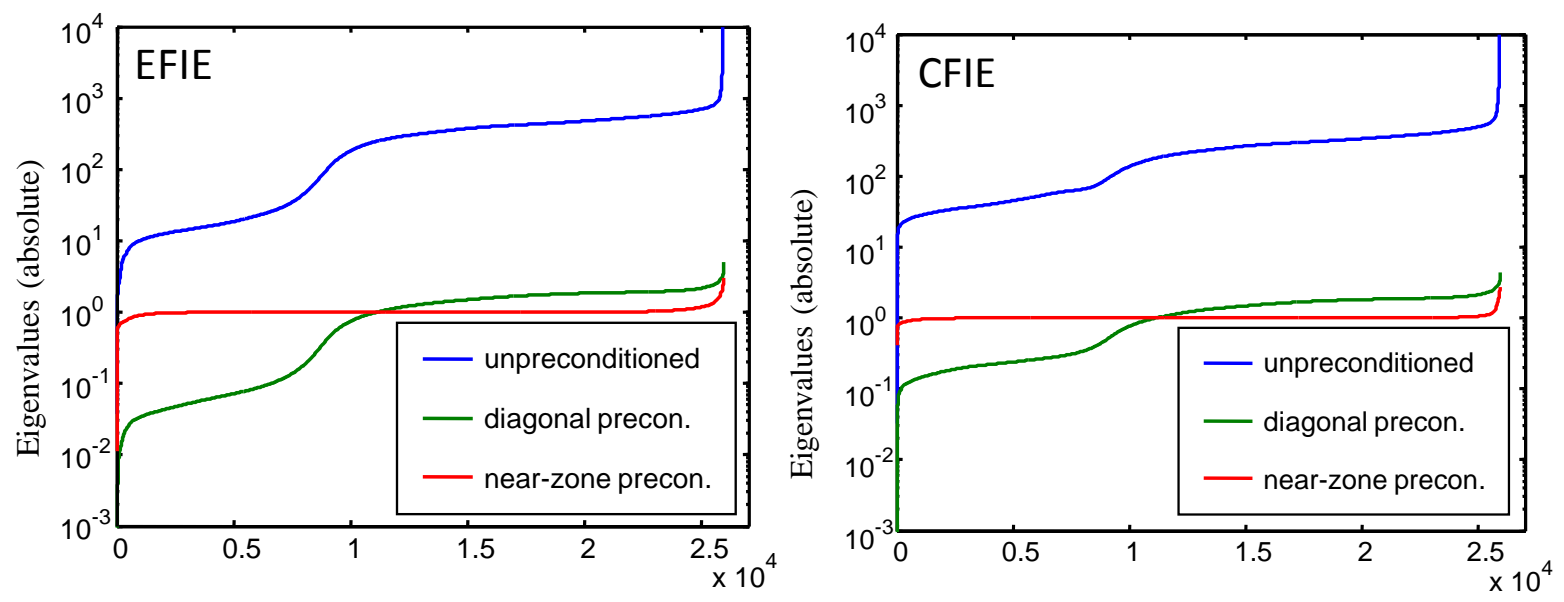

Fig. 3. Eigenvalue spectra of the problem considered in Fig. 2 for EFIE and CFIE case. The eigenvalues of the unpreconditioned system are compared with those of a diagonally and a LU preconditioned system.

convergence perceptibly better than the weak near-zone preconditioners. If the same object is considered at $100 \mathrm{GHz}$ and a discretization with 1091335 unknowns (Fig. 1, right plot), then it becomes clear that the benefit of the LU preconditioner is diminishing. This is due to the fact that the second term on the left hand side of Eq. (7) is getting crucial for the convergence.

In contrast to this, the near-zone LU preconditioner has only low impact on the convergence characteristics of the CFIE, which is naturally better conditioned than the EFIE, as described above. The convergence properties of the EFIE and the CFIE are compared by computing the RCS of an airplane (Fig. 2) at a frequency of $150 \mathrm{MHz}$. For this comparatively small problem with 25923 unknowns, the LU preconditioner improves the convergence considerably in the EFIE test case. It can be followed that for such a problem size, the preconditioner achieves a regularization of the EFIE so that the convergence behavior is similar to that of the CFIE. Furthermore, it becomes clear that the convergence of the CFIE depends only weakly on the particular kind of near-zone preconditioner, since the second-kind integral operator already leads to a well-conditioned system.

In order to get a deeper understanding of the different properties of first- and second-kind Fredholm integral operators, the complete spectrum of the system matrix of the problem considered in Fig. 2 is computed and illustrated in Fig. 3. The absolute eigenvalues of the original MoM matrix, the diagonally and the near-zone preconditioned system are plotted for the EFIE (left) and the CFIE (right). It can be discerned that the diagonal preconditioner has a normalizing effect and only shifts the spectra, while the near-zone preconditioner clusters all eigenvalues around one. This clearly demonstrates that the near-zone preconditioner transforms the integral operator in a way that it has the same spectral properties as a second-kind integral operator. For larger-scale problems, however, the near-zone preconditioner loses this

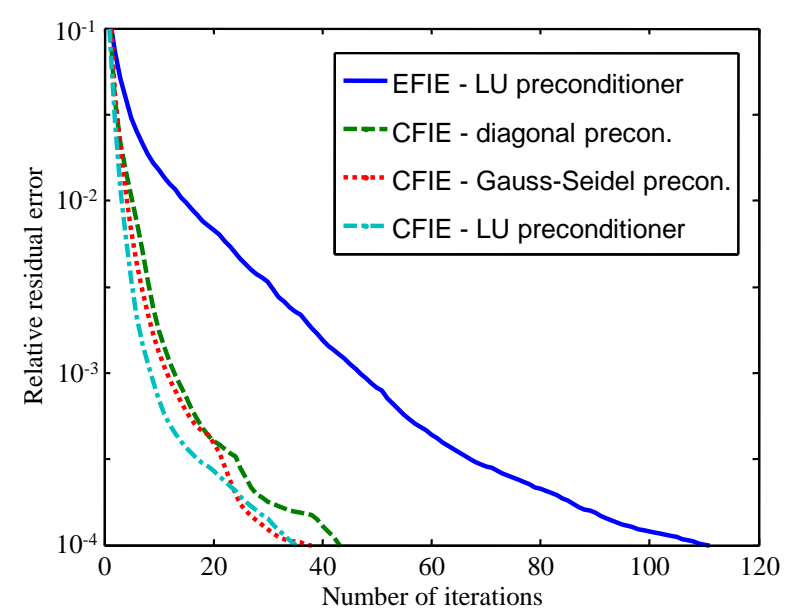

Fig. 4. Relative residual error for scattering computations of an air plane with 872736 unknowns.

effect, since the term $\mathbf{Z}_{\text {near }}^{-1} \mathbf{Z}_{\text {far }}$ in Eq. (7) is dominating the convergence behavior. By comparing the spectra of the EFIE and the CFIE, it can be discerned that both spectra are similar, but the identity operator of the CFIE helps to compress the spectrum closer around one, which is the mathematical explanation for the better convergence of the linear system derived from the CFIE.

Finally, the convergence characteristics of EFIE and CFIE are compared using a larger example. The airplane, illustrated in Fig. 2, is discretized with 872736 unknowns for a frequency of $900 \mathrm{MHz}$. It can be noticed in Fig. 4 that the near-zone preconditioner is no longer able to improve the spectrum of the EFIE in a way that the convergence is similar to CFIE computations. On the other hand, it becomes clear that the convergence of the CFIE depends only weakly on the particular kind of near-zone preconditioner. 


\section{Conclusions}

The effectiveness of near-zone preconditioning methods for the solution of integral equations has been investigated. It was shown that near-zone preconditioners can improve the convergence behavior of first-kind integral equations such as the EFIE considerably. A theoretical explanation was given by inspecting the eigenvalue spectrum of the EFIE and the CFIE operators. Moreover, it was demonstrated that the effect of near-zone preconditioners on the EFIE is decreasing for growing problem size. Furthermore, it can be concluded that preconditioners based on the near-field matrix only have a weak impact on second-kind integral operators like the CFIE independent from problem size.

\section{References}

Andriulli, F. P., Cools, K., Bağci, H., Olyslager, F., Buffa, A., Christiansen, S. H. and Michielssen, E.: A Multiplicative Calderón Preconditioner for the Electric Field Integral Equation, IEEE T. Antenn. Propag., 56, 2398-2412, doi:10.1109/TAP.2008.926788, 2008.

Buffa, A. and Christiansen, S. H.: A dual finite element complex on the barycentric refinement, Math. Comput., 76, 1743-1769, doi:10.1090/S0025-5718-07-01965-5, 2007.
Coifman, R., Rokhlin, V., and Wandzura, S.: The Fast Multipole Method for the Wave Equation: A Pedestrian Prescription, IEEE T. Antenn. Propag., 35, 7-12, doi:10.1109/74.250128, 1993.

Eibert, T. F.: Some Scattering Results Computed by SurfaceIntegral-Equation and Hybrid Finite-Element-Boundary-Integral Techniques, Accelerated by the Multilevel Fast Multipole Method, IEEE Antenn. Propag. M., 49, 61-69, doi:10.1109/MAP.2007.376638, 2007.

Rao, S. M., Wilton, D. R., and Glisson, A. W.: Electromagnetic Scattering by Surfaces of Arbitrary Shape, IEEE T. Antenn. Propag., 30, 409-418, doi:10.1109/TAP.1982.1142818, 1982.

Saad, Y.: A flexible inner-outer preconditioned GMRES algorithm, SIAM J. Sci. Comput., 14, 461-469, doi:10.1137/0914028, 1993.

Wiedenmann, O. and Eibert, T. F.: An Investigation of Near-Zone Preconditioning Techniques for Integral Equation Solutions by Method of Moments, International Conference on Electromagnetics in Advanced Applications (ICEAA), Torino, Italy, 12-16 September 2011, 199-202, 2011.

Wiedenmann, O. and Eibert, T. F.: Near-Zone Preconditioning Investigations for Integral Equation Solutions by Method of Moments, The 28th International Review of Progress in Applied Computational Electromagnetics, Columbus, Ohio, 10-14 April 2012, 427-432, 2012.

Yan, S., Jin, J.-M., and Nie, Z.: Improving the Accuracy of the Second-Kind Fredholm Integral Equations by Using the BuffaChristiansen Functions, IEEE T. Antenn. Propag., 59, 12991310, doi:10.1109/TAP.2011.2109364, 2011. 